\title{
Photostability enhancement of anionic natural dye by intercalation into hydrotalcite
}

\author{
Yoshiumi Kohno, ${ }^{*}, a$ Koichi Totsuka, ${ }^{a}$ Shuji Ikoma, ${ }^{b}$ Keiko Yoda, ${ }^{c}$ Masashi Shibata, ${ }^{c, d}$ \\ Ryoka Matsushima, ${ }^{a}$ Yasumasa Tomita, ${ }^{a}$ Yasuhisa Maeda, ${ }^{a}$ Kenkichiro Kobayashi ${ }^{a}$ \\ ${ }^{a}$ Department of Materials Science and Chemical Engineering, Faculty of Engineering, Shizuoka \\ University, 3-5-1 Johoku, Naka-ku, Hamamatsu, Shizuoka, 432-8561, Japan \\ ${ }^{b}$ Division of Applied Science and Chemical Engineering, Faculty of Engineering, Shizuoka University, \\ 3-5-1 Johoku, Naka-ku, Hamamatsu, Shizuoka, 432-8561, Japan \\ ${ }^{c}$ Beauty Research Center, Kao Corporation, 2-1-3 Bunka, Sumida-ku, Tokyo 131-8501, Japan \\ ${ }^{d}$ School of Bioscience and Biotechnology, Tokyo University of Technology, 1404 Katakura, Hachioji, \\ Tokyo 192-0982, Japan \\ * Corresponding author. E-mail: tykouno@ipc.shizuoka.ac.jp; Tel: +81-53-478-1623
}


2

3

\section{Abstract}

The aim of this study is the improvement of the photostability of several natural anionic dyes, carmine (CM), carthamus yellow (CY) and annatto dye (ANA), by complexation with hydrotalcite. The composite of the dyes and hydrotalcite is prepared by the co-precipitation method. CM is successfully intercalated in the hydrotalcite layer when the amount of introduced CM is large. The photostability of CM in CM/HT composite is superior to the CM adsorbed on silica surface. The effect of the stability enhancement is larger when the amount of introduced CM exceeds 0.23 g/g-host, or when the layer charge density of the hydrotalcite is larger. CY is also stabilized by complexation with hydrotalcite, whereas ANA is not stabilized by complexation with hydrotalcite. The photostability of an anionic natural dye can be improved by intercalation into the hydrotalcite layer, if the dye has hydrophilic nature and a rather planar structure. The intercalated dye is stabilized by the protection from the attack of the atmospheric oxygen. In addition, contribution of the electrostatic interaction between the positively charged hydrotalcite layer and the intercalated anionic dye is also proposed.

\section{Graphical abstract}

Anionic natural dyes intercalated between the layers of hydrotalcite are stabilized against irradiation. 
3 Keywords: anionic natural dye; hydrotalcite; layered double hydroxide; photostability;

4 stabilization; intercalation 


\section{Introduction}

Natural dyes are generally superior in safety than synthetic ones. However, their stability is not satisfactory for practical use as a colorant. Therefore, utilization of the natural dyes is limited only in the areas where particular safety is required, such as food or cosmetics. If the stability of these natural dyes is improved, they can be widely utilized as a general colorant.

Incorporation of organic dyes into inorganic hosts sometimes enhances the stability. For example, cationic dyes have been stabilized by intercalation between the layers of cation exchangeable clays [1-4] or zeolites [5-9]. We have also reported that the stability of naturally occurring anthocyanin dye is greatly enhanced by complexation with cation exchangeable clay, montmorillonite [10].

Hydrotalcite is one of natural minerals which exhibits anion exchange property. The carbonate ion in the interlayer can be substituted by other anions, including organic anionic dyes. For example, indigo carmine dye has been intercalated between the layers of the hydrotalcite [11]. Especially, attempts to immobilize [12] or stabilize [13-15] adsorbed organic azo dyes by complexation with hydrotalcite or hydrotalcite-like compound should be paid attention. On the other hand, it has been reported that intercalation of a perylene derivative into the hydrotalcite brings about no stabilization effect [16]. It has also been reported that betalain, which is one of natural anionic dyes, is adsorbed on hydrotalcite, but the dye is not intercalated and is not sufficiently stabilized on hydrotalcite compared to alumina[17]. 
The aim of this study is to enhance the stability of the anionic natural dyes by formation of the composite with hydrotalcite. As the anionic natural dyes, carmine, carthamus yellow and annatto dye are applied. These dyes have been considered less toxic and utilized as a food colorant $[18,19]$, but it has been pointed out that irradiation of these natural dyes may cause the occurrence of toxicity [20]. Therefore, in this study we check the enhancement of the stability of these dyes under irradiation, and explore the cause of the stabilization. The difference in the stabilization effect of the respective dyes is related to the differences in the molecular properties of these dyes.

\section{Material and methods}

The natural dyes used in this study were commercially available ones. Carmine (CM) and annatto dye (ANA) were supplied from Wako Chemical, whereas carthamus yellow (CY) was from Kanto Chemical. Scheme 1 represents the structural formulae of the main components included in the respective dyes, carminic acid, norbixin and safflomin.

Commercially available hydrotalcite (HT) was supplied from Wako Chemical. Silica (SIO) was supplied from Catalysis Society of Japan (JRC-SIO-6).

In general, hydrotalcite consists of carbonate ion as an interlayer anion, whose affinity to the hydrotalcite layer is very high. Hence, in spite of a previous report [21], the exchange of the carbonate by other anion is thought to be difficult in a conventional manner. In fact, the present dyes were not efficiently adsorbed and incorporated into HT 
by simply mixing HT and an aqueous solution of each dye. Therefore, we followed the so-called co-precipitation method [22, 23] as described below.

Each of $\mathrm{MgCl}_{2} \cdot 6 \mathrm{H}_{2} \mathrm{O}$ and $\mathrm{AlCl}_{3} \cdot 6 \mathrm{H}_{2} \mathrm{O}$, both supplied from Wako Chemical, was dissolved into deionized water and mixed at the ratio $x=\mathrm{Al} /(\mathrm{Mg}+\mathrm{Al})$ to be $0.20,0.25$, 0.30 or 0.33 , where total amount of cations $\left(\mathrm{Mg}^{2+}\right.$ and $\left.\mathrm{Al}^{3+}\right)$ was fixed to $25 \mathrm{mmol}$ [24].

Unless otherwise denoted, the value of $x$ was 0.30 . To the resulting mixture, an aqueous solution containing given amount of the dye was added. Then, the solution was mixed with $500 \mathrm{~cm}^{3}$ of a $0.1 \mathrm{~mol} / \mathrm{dm}^{3} \mathrm{NaOH}$ aqueous solution under $\mathrm{N}_{2}$ bubbling, and the $\mathrm{pH}$ was adjusted to 9.5. Total volume of the resulting solution was about $1000 \mathrm{~cm}^{3}$. The mixture was sealed, kept at $363 \mathrm{~K}$ for $24 \mathrm{~h}$, and then filtered. The filtrate was non-colored, indicating that all introduced dye was adsorbed on HT. The sediment was collected, washed with water and dried at $323 \mathrm{~K}$. Thus obtained composite samples are denoted as Dye $(w) / H T$, where Dye is the synonym of the dye and $w$ indicates the weight of introduced dye per HT (g/g-HT).

To investigate the saturated amount of adsorbed CM onto HT, the amount of introduced CM was increased to exceed the adsorption maximum, so that the filtrate remains colored to some extent. The amount of adsorbed CM was calculated from the difference in the absorbance of the original CM solution and of the filtrate.

On the other hand, the natural dyes (CM, CY and ANA) were not adsorbed spontaneously onto SIO, because the surface of SIO was not positively charged as HT. Therefore, the composites of SIO and the dyes (Dye/SIO) were prepared by mixing the aqueous dye solution (20 mg/50 ml) and SIO (500 mg), followed by evaporation of the 
1$$
5
$$

solvent at $343 \mathrm{~K}$.

In addition, intimately ground physical mixture of CM and the commercial HT was prepared as the reference sample $(\mathrm{CM}+\mathrm{HT})$. The weight ratio of $\mathrm{CM}$ and $\mathrm{HT}$ was determined so that the absorption of the mixture at $\lambda_{\max }$ became about 1 .

XRD patterns were measured with the Rigaku MiniFlex X-ray diffractometer (CuK $\alpha$, step angle and scan speed $0.05^{\circ}$ and $2^{\circ} / \mathrm{min}, 15 \mathrm{~mA}, 30 \mathrm{kV}$ ).

UV-Vis spectra were collected with the JASCO V-550 spectrophotometer. To measure diffuse-reflectance spectra, the ISV-469 integrating sphere was equipped to the spectrometer.

To investigate the light endurance of each sample, visible light was irradiated to the samples under ambient temperature and pressure. The color fading was measured by the decrease of the absorption in the diffuse reflectance spectra. In order to set the light absorption within the ranges of the spectrophotometer, the samples were mixed and diluted with the commercial HT. A 100 W halogen lamp (Schott Megalight 100) equipped with a $30 \mathrm{~cm}$ length flexible light guide was used as a visible light source. The powder samples were loaded in a plastic cell covered with a thin glass, and placed at the position of $2 \mathrm{~cm}$ from the aperture of the light guide. When the sample was irradiated under $\mathrm{N}_{2}$ atmosphere, the sample cell was packed in a transparent gas barrier film filled with nitrogen.

\section{Results and discussion}




\subsection{Composite sample of CM and HT}

3

Figure 1 illustrates the XRD patterns of CM/HT samples together with pure HT. The

5 pure HT showed a sharp peak at $11.6^{\circ}$, corresponding to the basal spacing of $0.76 \mathrm{~nm}$. As the amount of introduced CM increased and $w$ exceeded 0.23 , the original peak was weakened and another diffraction peak appeared at lower angle around $7.2^{\circ}$, corresponding to the basal spacing of $1.23 \mathrm{~nm}$. This indicates that the interlayer distance increased by introducing large amount of CM. In Naphthol Yellow S/hydrotalcite system, it has been reported that similar change in the XRD pattern has been observed by the change in the amount of introduced dye [25]. As the thickness of the HT layer is about $0.48 \mathrm{~nm}$, the interlayer distance is calculated to be $0.75 \mathrm{~nm}$. This value is roughly coincides with the molecular thickness of CM $(0.55 \mathrm{~nm})$. In addition, no elution of CM was observed when the $\mathrm{CM} / \mathrm{HT}$ sample was washed with water. From these results, we concluded that CM was intercalated in the interlayer space of HT, especially when the amount of introduced CM was large.

$$
\text { --- (Figure 1) --- }
$$

The peak corresponding to the increased interlayer distance in CM/HT seemed rather broad. This implies that the intercalation of CM into HT did not uniformly occur, and that the structure of CM/HT was disordered. The irregularity of the structure has often observed in the hydrotalcite including bulky organic molecules [16, 21].

Table 1 shows the saturated amount of adsorbed CM on various HT samples having 
different layer charges. Although there were some difference between the ideal and real values, the layer charge of HT could be related to the maximum adsorption amount of CM. Considering this, it can be said that at least main part of CM was not physically adsorbed on the surface of HT but immobilized in the interlayer of HT by anion exchange.

--- (Table 1) ---

\subsection{Spectra of $\mathrm{CM} / \mathrm{HT}$}

Figure 2 illustrates the diffuse reflectance UV-vis spectra of CM/HT with various amount of CM. In all samples a peak with a shoulder was observed in the visible light region of $\lambda=520 \mathrm{~nm}$ to $570 \mathrm{~nm}$. This absorption is assigned to that of $\mathrm{CM}$, indicating that CM was not decomposed by the operation to form the composite with HT.

$$
\text { --- (Figure 2) --- }
$$

When the amount of introduced CM increased, predominant growth of the peak at longer wavelength around $568 \mathrm{~nm}$ was observed. The shift to the longer wavelength is not inconsistent to the expectation of the electrostatic interaction between the HT layer and CM [26], together with the co-planarization of $\mathrm{CM}$ molecule in the narrow interlayer space of HT [27]. In addition, there still remains the possibility that the peak shift was caused by the formation of J-aggregate of CM between the HT layers.

\subsection{Enhancement of light fastness}


The CM/HT samples were exposed to visible light irradiation and the spectral changes

3

were traced. Along with the irradiation, the absorption in the visible region decreased and no other changes in the spectra were observed, indicating the simple decomposition of CM under irradiation. The changes in the absorption of several CM composite samples during irradiation were illustrated in Figure 3. The photostability of CM/SIO could be judged to be low, because only $55 \%$ of absorption was maintained after 150 min of irradiation. $\mathrm{CM}(0.025) / \mathrm{HT}$ showed nearly the same level of stability (61 \%) as CM/SIO. Therefore, it seems that no stabilization effect was brought about by the complexation with $\mathrm{HT}$ in $\mathrm{CM}(0.025) / \mathrm{HT}$. However, $\mathrm{CM}(1.85) / \mathrm{HT}$ exhibited very high durability against visible light irradiation (91\%). This result suggested that the CM/HT composite showed enhanced photostability of CM when large amount of CM was introduced. On the other hand, no such stabilization effect was seen in the physical mixture of $\mathrm{CM}$ and $\mathrm{HT}(\mathrm{CM}+\mathrm{HT})$. The $\mathrm{CM}$ molecule in the $\mathrm{CM}+\mathrm{HT}$ sample was expected to be highly aggregated, because the original CM powder was not molecularly dispersed but simply mixed with $\mathrm{HT}$ in $\mathrm{CM}+\mathrm{HT}$. Therefore, the enhancement of the durability against irradiation did not come simply from the high concentration (i. e., aggregation) of CM, although other study has been claimed that aggregation of azo-dye in the interlayer of LDH enhances the stability [28].

$$
\text { --- (Figure 3) --- }
$$

Figure 4 represents the relationship between the photostability and the amount of adsorbed $\mathrm{CM}$ in the $\mathrm{CM}(w) / \mathrm{HT}$ samples. The stabilization effect was enhanced as the 
CM amount increased up to $w=0.25$, whereas drastic improvement was not observed when $w$ was greater than 0.25 . On the other hand, as shown in Figure 1, well defined peak corresponding to the increase in the interlayer space came to appear when $w$ exceeded 0.23. Therefore, it is natural to think that the enhancement of the photostability of $\mathrm{CM}$ in $\mathrm{CM} / \mathrm{HT}$ is in relation to the increase in the interlayer space of HT in CM/HT. As the increase in the interlayer space means the intercalation of CM into the HT layer, it can be concluded that the intercalation brought about the enhancement of photostability.

\section{--- (Figure 4) ---}

\subsection{Cause of the stability enhancement}

The occurrence of singlet oxygen has been thought to be one of the main causes in degradation of natural dyes under irradiation [29, 30]. Table 2 represents the durability of CM composite samples against irradiation in the presence or absence of oxygen. As explained above, $\mathrm{CM}(0.025) / \mathrm{HT}$ did not exhibit enough stability in the air. However, when the sample was maintained under $\mathrm{N}_{2}$ to prevent the contact of atmospheric oxygen, even CM(0.025)/HT sample showed high stability. Furthermore, CM/SIO sample also showed high stability against irradiation under $\mathrm{N}_{2}$ atmosphere. This result suggests that photodecomposition of CM in CM/HT was due to the oxidation by atmospheric oxygen. Therefore, the stability enhancement of CM/HT with increased amount of CM should be considered that the attack of molecular oxygen from the air is prevented when CM is 
intercalated between the HT layers.

$$
\text { --- (Table 2) --- }
$$

Table 3 represents the difference in the light fastness of $\mathrm{CM}(0.25) / \mathrm{HT}$ when the layer charge of HT was changed. Although the difference in the durability was rather small, the order of the durability was identical to the order of the layer charge density. Since the amount of CM was set to be the same among these samples, it can be concluded that the higher stabilization effect was obtained as the layer charge was larger. This result suggests the possibility that the electrostatic interaction between CM and HT layer also contributes to the stabilization of $\mathrm{CM}$ in $\mathrm{CM} / \mathrm{HT}$. However, the stability enhancement mentioned here could not be ascribed only to the electrostatic interaction, because the change in the layer charge density would influence many properties of HT such as interlayer distance.

$$
\text { --- (Table 3) --- }
$$

From these results, we concluded that the stabilization effect of $\mathrm{CM}$ in $\mathrm{CM} / \mathrm{HT}$ is brought about by the defense of CM from the attack of atmospheric oxygen. In addition, there is a possibility that the stability of CM is enhanced by the electrostatic effect from the positive charge of the HT layer.

\subsection{Stabilization of other natural dyes}

We then tried to confirm the enhancement of the stability of CY and ANA as natural dyes other than CM. As shown in Table 4, the stability of CY/HT was higher than 
CY/SIO. Therefore, CY was stabilized by complexation with HT. As CM does, CY has a planar molecular structure and shows hydrophilic nature caused by a sugar moiety, so that CY is expected to show affinity to the interlayer space. Since these characters are similar to those of CM, it can be concluded that CY is stabilized in the interlayer of HT by the same reason as $\mathrm{CM} / \mathrm{HT}$.

$$
\text { --- (Table 4) --- }
$$

On the other hand, the durability of ANA/HT was the same level as ANA/SIO, and the stabilization effect was not observed by complexation with HT, as shown in Table 4. In the XRD pattern of ANA/HT, no peak shift toward lower angle was observed even when the amount of ANA was increased to the adsorption maximum. Contrary to CM and CY, ANA has a rather linear structure and shows hydrophobic character. In fact, when ANA/HT sample was washed with ethanol, almost all ANA was eluted easily. This result indicated that ANA was adsorbed on the external surface of HT by hydrophobic interaction. Hence, we concluded that the stabilization effect of ANA was not observed in ANA/HT because ANA was not included between the HT layers. Organomodification of the interlayer space of HT [31, 32] may be effective to intercalate hydrophobic dye [33] such as ANA, resulting in the stabilization of the dye. In conclusion, the photostability of an anionic natural dye can be improved by intercalation into the HT layer, if the dye has hydrophilic nature and a rather planar structure. The dye intercalated in the HT layer is stabilized by the protection from the attack of the atmospheric oxygen. However, contribution of the electrostatic interaction from the positively charged HT layer should also be considered. 


\section{References}

[1] T. Endo, N. Nakada, T. Sato, M. Shimada, J. Phys. Chem. Solids 50 (1989) 133.

[2] T. Shichi, K. Takagi, J. Photochem. Photobiol. C 1 (2000) 113.

[3] T. Saito, K. Fukui, Y. Kodera, A. Matsuyama, H. Nishimura, Y. Inada, Dyes Pigm. 65 (2005) 21.

[4] Y. Kohno, R. Hoshino, R. Matsushima, Y. Tomita, K. Kobayashi, J. Jpn. Soc. Colour Mater. 80 (2007) 6.

[5] R. Hoppe, G. Schulz-Ekloff, D. Woehrle, C. Kirschhock, H. Fuess, Stud. Surf. Sci. Catal. 84 (1994) 821.

[6] D. Wöhrle, A.K. Sobbi, O. Franke, G. Schulz-Ekloff, Zeolites 15 (1995) 540.

[7] G. Calzaferri, D. Bruhwiler, S. Megelski, M. Pfenniger, M. Pauchard, B. Hennessy, H. Maas, A. Devaux, U. Graf, Solid State Sci. 2 (2000) 421.

[8] Z. Gabelica, S. Valange, M. Shibata, H. Hotta, T. Suzuki, Microporous Mesoporous Mater. 44-45 (2001) 645.

[9] Y. Kohno, Y. Shibata, N. Oyaizu, K. Yoda, M. Shibata, R. Matsushima, Microporous Mesoporous Mater. 114 (2008) 373.

[10] Y. Kohno, R. Kinoshita, S. Ikoma, K. Yoda, M. Shibata, R. Matsushima, Y. Tomita, Y. Maeda, K. Kobayashi, Appl. Clay Sci. 42 (2009) 519.

[11] L. El Gaini, M. Lakraimi, E. Sebbar, A. Meghea, M. Bakasse, J. Hazard. Mater. 161 (2009) 627.

[12] H. Laguna, S. Loera, I.A. Ibarra, E. Lima, M.A. Vera, V. Lara, Microporous Mesoporous Mater. 98 (2007) 234.

[13] M.Z.b. Hussein, Z. Zainal, A.H. Yahaya, A.b.A. Aziz, Mater. Sci. Eng. B 88 (2002) 98.

[14] S. Guo, D. Li, W. Zhang, M. Pu, D.G. Evans, X. Duan, J. Solid State Chem. 177 (2004) 4597.

[15] Y. Tian, G. Wang, F. Li, D.G. Evans, Mater. Lett. 61 (2007) 1662.

[16] J. Bauer, P. Behrens, M. Speckbacher, H. Langhals, Adv. Funct. Mater. 13 (2003) 241.

[17] E. Lima, P. Bosch, S. Loera, I.A. Ibarra, H. Laguna, V. Lara, Appl. Clay Sci. 42 (2009) 478.

[18] F.E. Lancaster, J.F. Lawrence, J. Chromatogr. A 732 (1996) 394. 
1 [19] S. Kawazoe, Y. Hojo, T. Mizutani, J. Health Sci. 46 (2000) 56.

2 [20] S. Arimoto-Kobayashi, M. Machida, K. Okamoto, A. Yamaguchi, Mutagenesis 20 (2005) 229.

$4 \quad$ [21] A.R. Auxilio, P.C. Andrews, P.C. Junk, L. Spiccia, D. Neumann, W. Raverty, N. Vanderhoek, Polyhedron 26 (2007) 3479.

6 [22] T.S. Stanimirova, G. Kirov, E. Dinolova, J. Mater. Sci. Lett. 20 (2001) 453.

7 [23] Y. Kubo, M. Tsuji, J. Jpn. Soc. Colour Mater. (in Japanese) 69 (1996) 667.

$8 \quad$ [24] S. Miyata, Clays Clay Miner. 28 (1980) 50.

$9 \quad$ [25] S. Miyata, Clays Clay Miner. 31 (1983) 305.

[26] M. Ogawa, R. Kawai, K. Kuroda, J. Phys. Chem. 100 (1996) 16218.

11 [27] M. Eguchi, S. Takagi, H. Inoue, Chem. Lett. 35 (2006) 14.

[28] P. Aranyosi, Z. Csepregi, I. Rusznák, L. Töke, A. Víg, Dyes Pigments 37 (1998) 33.

[29] H. Oda, Color. Technol. 117 (2001) 204.

[30] H. Oda, Color. Technol. 117 (2001) 257.

[31] K. Inomata, M. Ogawa, Bull. Chem. Soc. Jpn. 79 (2006) 336.

[32] Y. Kameshima, H. Yoshizaki, A. Nakajima, K. Okada, J. Colloid Interface Sci. 298 (2006) 624.

[33] N. Kakegawa, M. Ogawa, Appl. Clay Sci. 22 (2002) 137. 


\section{$1 \quad$ Figure caption}

2 Figure 1. XRD patterns of HT (upper curve) and CM(1.85)/HT (lower curve). The inset 3 indicates the XRD patterns of CM/HT samples with various amounts of CM. (a) $4 \mathrm{CM}(0.025) / \mathrm{HT}$, (b) $\mathrm{CM}(0.1) / \mathrm{HT}$, (c) $\mathrm{CM}(0.23) / \mathrm{HT}$, (d) $\mathrm{CM}(0.46) / \mathrm{HT}$, (e) $\mathrm{CM}(0.92) / \mathrm{HT}$ 5 and (f) $\mathrm{CM}(1.85) / \mathrm{HT}$, respectively.

Figure 2. Diffuse reflectance UV-Vis spectra of $\mathrm{CM}(0.025) / \mathrm{HT}$ (dotted line), normalized at the maximum value. 


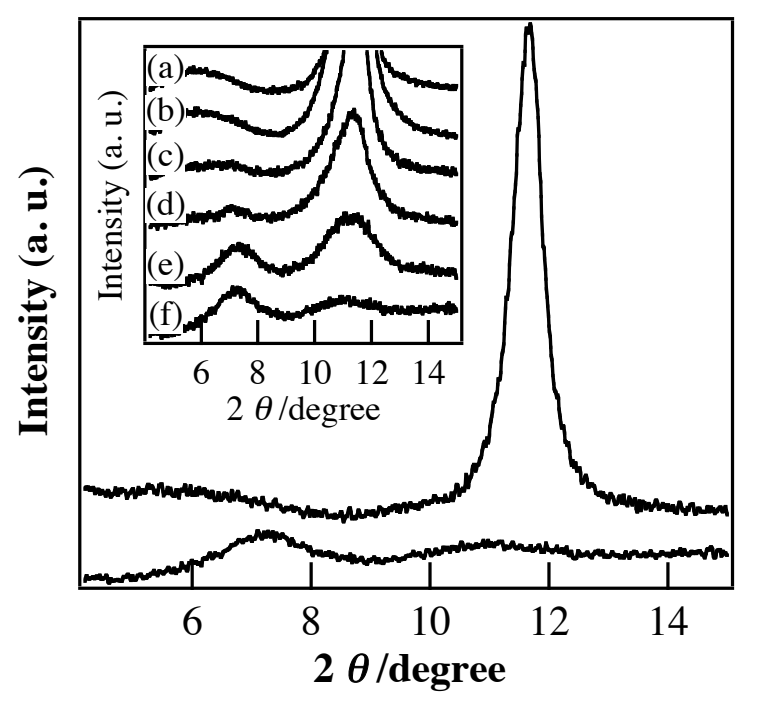

Figure 1 XRD patterns of HT (upper curve) and CM(1.85)/HT (lower curve). The inset indicates the XRD patterns of CM/HT samples with various amounts of CM. (a) $\mathrm{CM}(0.025) / \mathrm{HT}$, (b) $\mathrm{CM}(0.1) / \mathrm{HT}$, (c) $\mathrm{CM}(0.23) / \mathrm{HT}$, (d) $\mathrm{CM}(0.46) / \mathrm{HT}$, (e) $\mathrm{CM}(0.92) / \mathrm{HT}$ and (f) $\mathrm{CM}(1.85) / \mathrm{HT}$, respectively. 


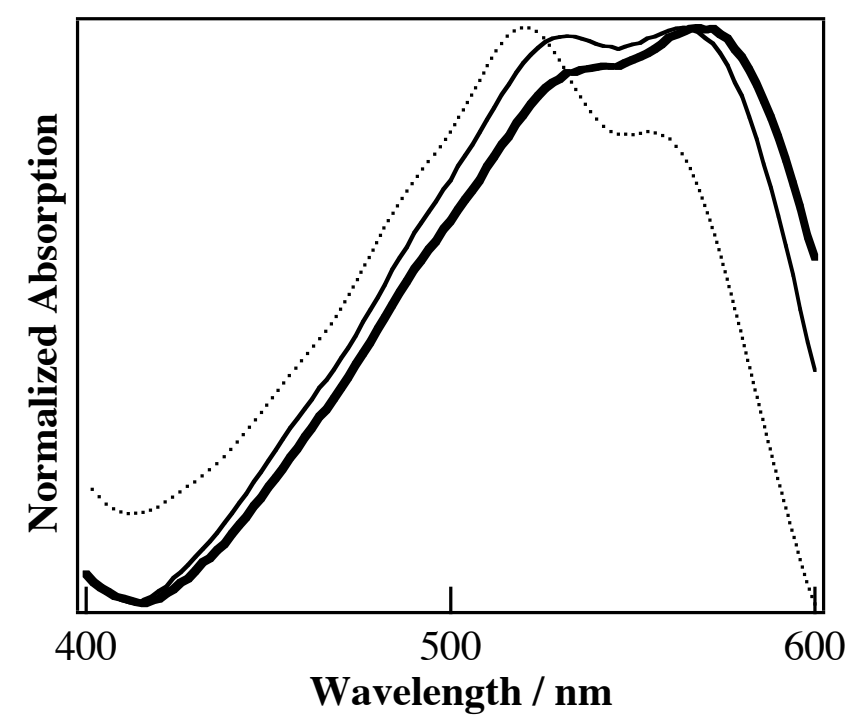

Figure 2 Diffuse reflectance UV-Vis spectra of $\mathrm{CM}(0.025) / \mathrm{HT}$ (dotted line), $\mathrm{CM}(0.46) / \mathrm{HT}$ (thin line) and $\mathrm{CM}(1.85) / \mathrm{HT}$ (thick line). The ordinate values are normalized at the maximum value. 


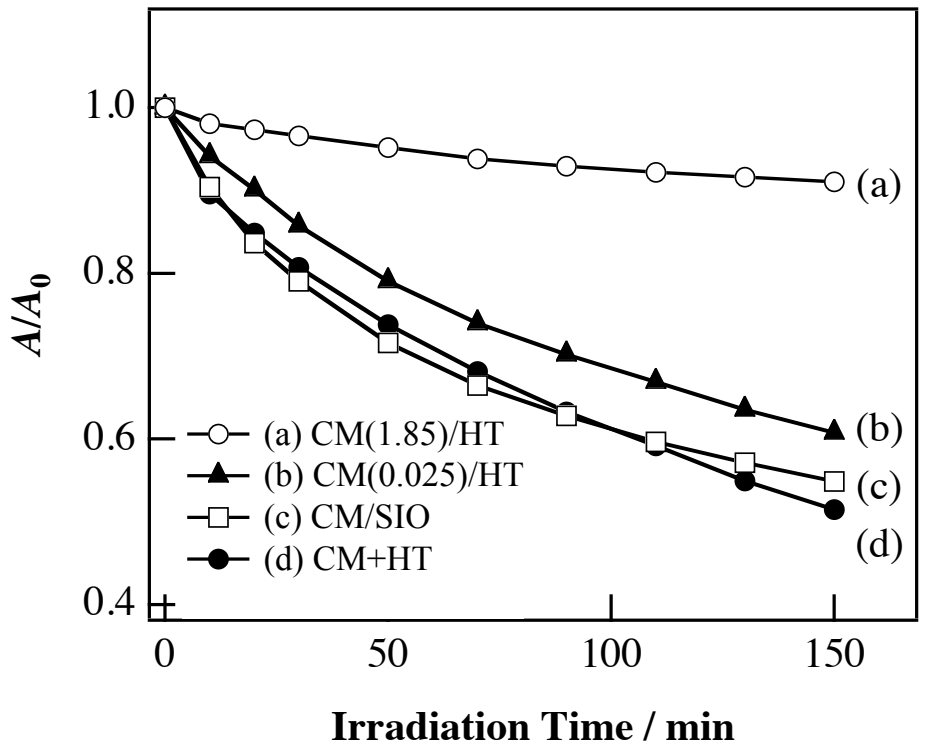

Figure 3 Changes in the absorption of several CM composites during irradiation for $150 \mathrm{~min}$. (a) $\mathrm{CM}(1.85) / \mathrm{HT}$, (b) $\mathrm{CM}(0.025) / \mathrm{HT}$, (c)

$\mathrm{CM} / \mathrm{SIO}$ and (d) $\mathrm{CM}+\mathrm{HT}$, respectively. 


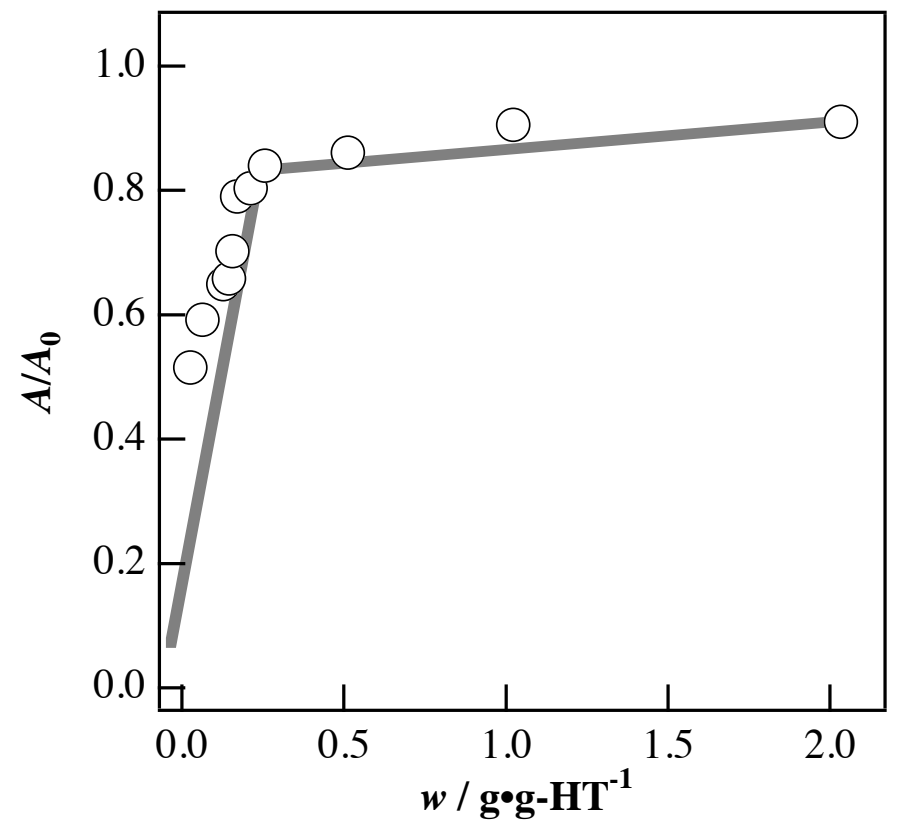

Figure 4 Relationship between the photostability against visible light irradiation and the amount of adsorbed CM in the CM/HT samples. 


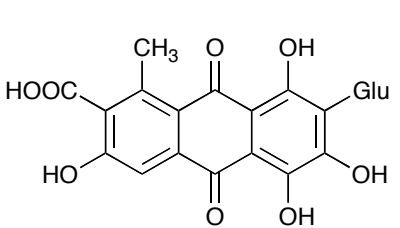

carminic acid

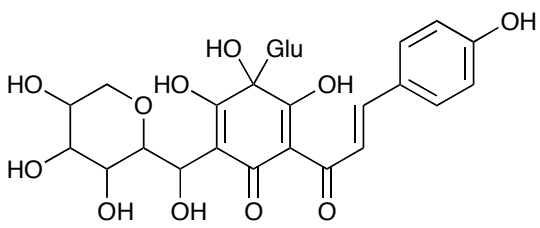

carthamus yellow<smiles>CC(/C=C/C=C(C)/C=C/C(=O)O)=C\C=C\C=C(C)\C=C\C=C(C)\C=C\C(=O)O</smiles>

norbixin

Scheme 1 